\title{
A Primary Malarial Infection Is Composed of a Very Wide Range of Genetically Diverse but Related Parasites
}

\author{
Pierre Druilhe, ${ }^{\star}$ Pierre Daubersies, ${ }^{\star}$ Jintana Patarapotikul, ${ }^{\S}$ Caroline Gentil, ${ }^{\star}$ Laurent Chene, ${ }^{\star}$ Tan Chongsuphajaisiddhi, \\ Sylvie Mellouk, ${ }^{*}$ and Gordon Langsley ${ }^{\ddagger}$ \\ $*$ Laboratory of Biomedical Parasitology, and ${ }^{\ddagger}$ Unit of Experimental Parasitology, Pasteur Institute, 75724 Paris Cedex 15 , France; and \\ ${ }^{\S}$ Department of Microbiology and ${ }^{\|}$Department of Pediatrics, Mahidol University, 10400 Bangkok, Thailand
}

\begin{abstract}
To address the question of how many distinct parasites are injected when a mosquito bites, we have characterized isolates resulting most probably from a single sporozoite inoculum. We describe the direct and immediate cloning on hepatocyte feeder layers of a Thai and an African Plasmodium falciparum primary isolate and the characterization of 67 independent clones by four techniques totaling nine different markers. This led to three main conclusions: $(a)$ both the phenotypic and genotypic markers revealed an unexpectedly large degree of diversity within the clones from a single isolate; (b) the clones are nonetheless genetically related; and $(c)$ a single mosquito inoculum would most likely be sufficient to generate considerable isolate complexity in the absence of repeated exposure. This diversity, which has been greatly underestimated in previous studies, does not bode well for the development of successful malaria control means. (J. Clin. Invest. 1998. 101:2008-2016.) Key words: characterization $\bullet$ parasite cloning $\bullet$ Plasmodium falciparum • polymorphism
\end{abstract}

\section{Introduction}

It has long been known that malaria clinical isolates are composed of a mixture of different parasite strains, and it is believed that this complexity is a severe drawback to successful malaria control. However, it has always been assumed that isolate polymorphism results from the superimposition of multiple infections by genetically distinct parasites (1-5). The contribution of a single mosquito inoculum to isolate polymorphism has never been estimated. Therefore, we sought clinical isolates derived from a single mosquito bite, reasoning that subsequent phenotyping and genotyping of cloned parasite lines derived from these "primary attack" isolates should reveal the complexity of a mosquito inoculum.

A detailed phenotypic and genotypic analysis of different cloned lines making up an isolate requires first the in vitro cultivation and dilution cloning of Plasmodium falciparum. How-

Address correspondence to Dr. Pierre Druilhe, Laboratoire de Parasitologie Biomedicale, Institut Pasteur, 28 rue du Dr. Roux, 75724 Paris Cedex 15, France. Phone: 33-1-4568-8578; FAX: 33-1-45688640; E-mail: druilhe@pasteur.fr

Received for publication 9 December 1996 and accepted in revised form 24 February 1998.

J. Clin. Invest.

(C) The American Society for Clinical Investigation, Inc. 0021-9738/98/05/2008/09 \$2.00

Volume 101, Number 9, May 1998, 2008-2016

http://www.jci.org ever, culture adaptation has long been known to introduce a strong selective pressure on the parasite population constituting an isolate $(6,7)$. To our knowledge, clonal analysis of isolates has been applied only to a Thai isolate (4) and to a limited extent to Sudanese samples (8) which had all been established for some time in culture. The very few clones characterized, combined with adaptation to in vitro culture, probably led to an underestimate of isolate diversity. The introduction of PCR allowed direct analysis of various parasite alleles in clinical isolates without the necessity of prior in vitro culture (1-3, 9-11). By this means, the allelic distribution for merozoite surface antigen 1 (MSP-1) ${ }^{1}$ and MSP-2 was shown to be different in wild parasites compared with laboratory-established strains, thus amply illustrating the influence of culture adaptation on isolate diversity $(3,7,12)$.

Isolate complexity varies over time, and depending on the clinical status of the individual, fluctuations in the circulating parasite population are detected in successive blood sampling (1, 13). However, PCR analysis of polymorphic alleles obtained from blood samples of patients living in an area of unstable malaria (low transmission) also revealed a marked degree of allelic complexity $(3,11)$. This argues that isolate polymorphism does not depend directly on the degree of malaria transmission (nor on the degree of immunity), and implies that the presence of multiple different genetic parasites within a single isolate might result not only from superinfection, but could also be due to infection by a single mix of genetically diverse parasites.

To test the above hypothesis, we used a relatively complex cloning procedure aimed at maintaining representation of the clonal content of our primary clinical isolates. Cloning was initiated immediately on the day of sampling, using hepatocytes as feeder layers, a technique which was formerly found capable of ensuring a consistent adaptation and faster speed of growth of newly sampled isolates (14). Parasite donors were selected from among patients with the greatest likelihood of having received a very limited, preferably a single, infected mosquito bite. Thereafter, these clones were characterized not only by PCR amplification of polymorphic loci, but also by their susceptibility to three different drugs as well as by karyotype, and by restriction fragment length polymorphism (RFLP) analysis. The results demonstrate a large degree of diversity within the clones, together with indications that these clones are nevertheless genetically related. These data imply that a single mosquito inoculum contains a large number of ge-

1. Abbreviations used in this paper: $\mathrm{CS}$, circumsporozoite; IFA, immunofluorescence assay; MSP, merozoite surface antigen; PFGE, pulsed field gradient electrophoresis; RBC, red blood cell; RFLP, restriction fragment length polymorphism; TRAP, thrombospondin-related anonymous protein. 
netically different parasites, most likely produced after genetic recombination within the mosquito. They also indicate that a single rather than multiple infection is sufficient to generate isolate complexity, one of the biggest challenges faced by malaria control and particularly by vaccine development.

\section{Methods}

Fast cloning procedure. Using strains already adapted to grow in standard culture conditions, we first developed a variable hematocrit procedure which, by requiring a single medium change every $3 \mathrm{~d}$ without removal of red blood cells (RBCs), enabled us to handle a large number of clones. The culture procedure followed is summarized in Table I. Starting from a very low hematocrit, 3/4 of spent medium was removed every $3 \mathrm{~d}$ without removing settled RBCs and was replaced by fresh medium containing fresh RBCs. Thus, the hematocrit increased from a starting $0.5 \%$ up to $4.6 \%$ over the first $15 \mathrm{~d}$. On day 15, the clones were subcultured as follows: using an eight-well multipipette, the spent medium and RBCs were resuspended, $150 \mu \mathrm{l}$ of cell suspension was removed, and $150 \mu$ l of fresh medium with fresh RBCs was added to the remaining $50 \mu$ l of cell suspension. The cell suspension removed was not discarded; it was used to create a "replica" plate which was subsequently used to detect positive wells. In this way, detection was done at different times only on replicas, without disturbing the growth of the master plate. Subsequent subcultures and other replicas were performed on days 21 and 27.

To allow for an early detection of positive wells and also to adjust precisely the parasite concentration in the suspension used for cloning, we also designed a direct immunofluorescence assay (IFA) detection method. When labeled by polyclonal antibodies, not only the parasite (here at the ring stage), but also the infected RBC itself becomes visible; therefore, wells can be screened under a magnification of 100 rather than $1,000.5 \mu \mathrm{l}$ of the RBC suspension at $3 \%$ hematocrit $\left(\sim 1.5 \times 10^{6} \mathrm{RBCs}\right)$ from replica plates was deposited onto a dried agarose film in 18-well microscope slides. Thereafter, a classical IFA assay was performed using whole human IgG from hyperimmune individuals as first antibody. The content of one well $(\sim 1.5 \times$ $10^{6} \mathrm{RBCs}$ ) could be screened in this way under $10 \times$ dry microscope objective within $\sim 2 \mathrm{~min}$; this is $\sim 10$ times faster than a classical Giemsa-stained thick smear for the same level of detection.

Table I. Amount of Medium and Fresh RBCs Added at Different Times

\begin{tabular}{lccccc}
\hline Date & $\begin{array}{c}\text { Medium } \\
\text { renewed }\end{array}$ & $\begin{array}{c}\text { RBCs } \\
\text { added }\end{array}$ & $\begin{array}{c}\text { Total RBCs } \\
\text { per well }\end{array}$ & Hematocrit & $\begin{array}{c}\text { Duplicate } \\
\text { plate }\end{array}$ \\
\hline & $\mu l$ & $\mu l$ & $\mu l$ & $\%$ & \\
D0 & 200 & 1 & 1 & 0.5 & \\
D3 & 150 & 0.7 & 1.7 & 0.8 & \\
D6 & 150 & 1.3 & 3 & 1.5 & \\
D9 & 150 & 2.3 & 5.3 & 2.6 & \\
D12 & 150 & 4 & 9.3 & 4.6 & $*$ \\
D15 & 150 & 2 & 4 & $\mathbf{2}$ & $*$ \\
D18 & 150 & 4 & 8 & 4 & $*$ \\
D21 & 150 & 2 & 4 & $\mathbf{2}$ & $*$ \\
D24 & 150 & 4 & 8 & 4 & $*$ \\
D27 & 150 & 2 & 4 & $\mathbf{2}$ & $*$ \\
D30 & 150 & 4 & 8 & 2 & \\
& & & & & $*$
\end{tabular}

On days 15, 21, and 27 (in bold), the cells were resuspended before removing $150 \mu \mathrm{l}$ of medium and adding the new medium and the fresh RBCs. Removed RBCs were used to prepare a duplicate.
To adjust precisely the cell suspension to yield an average of one parasite every two wells, the suspension was distributed on two IFA slides, and all 36 wells were screened by IFA. IFA slides were also prepared in between the distribution into each culture well, so as to check retrospectively that a statistical 0.5 parasite per well had indeed been distributed into each cloning well.

Finally, the procedure was tested by cloning culture-adapted strains, i.e., the Uganda Palo Alto (FUP/C) and the NF54 strains in standard culture conditions (RPMI 1640 medium supplemented with $10 \%$ human $\mathrm{AB}$ serum).

Selection of primary clinical isolates. Two approaches were used in order to gather $P$. falciparum isolates which would most likely result from a single infected mosquito bite. One was to select cases from very short duration travel, i.e., $<1 \mathrm{wk}$, of Europeans in an endemic area. It is known that among the approximately 20 million travelers from the North to malaria endemic regions, only a very small proportion will be infected. For instance, the risk was estimated recently to be one $P$. falciparum infection in 34,000 travelers from the United Kingdom, with a mean travel time of 4 wk (15). For shorter travel, i.e., $<1 \mathrm{wk}$, the chance of receiving two infective bites can be estimated to be statistically $<1 / 100,000$. Although no full guarantee can be obtained and no technique can ascertain that only one infected mosquito had bitten, this is extremely unlikely in statistical terms. The other approach, which follows the same type of reasoning, was applied to Thai isolates: malaria transmission is very heterogeneous in the country, where areas devoid of transmission coexist with areas of low transmission (in the order of one infected Anopheles per 6,000 noninfected) $(16,17)$, which indeed would yield a mean rate of one infective bite every $3 \mathrm{yr}$ as shown recently (18). Therefore, by selecting inhabitants of nonendemic regions of a low transmission country, contamination resulting from local travel would also yield an extremely low risk of multiple infected bites.

31 cases were selected according to the above criteria from individuals having an apparent primary $P$. falciparum attack. 23 were Europeans returning from Africa after a vacation of $<2 \mathrm{wk}$, and 8 were Thai subjects from a nonendemic district, with no previous history of malarial infection, who became infected upon travel to a malariaprone region. Thus, although there is no absolute certainty, the cases were selected to obtain the best probability estimates that we were dealing with single inoculation isolates, and so as to have the lowest grade of multiparasitism (i.e., as much as possible, one ring-stage per infected RBC).

Cloning of fresh isolates using hepatocyte feeder layers. In our first report (14), we obtained an improved growth, not only when hepatocytes were actually present together with parasitized RBCs, but also when spent medium from hepatocyte cultures was used to grow parasitized RBCs. The latter method would have been convenient for cloning; however, it proved suboptimal in promoting the growth of nonadapted isolates (Fig. 1). Consequently, we had to come back to the former, more laborious procedure using feeder cells.

Rat hepatocytes were prepared by collagenase dissociation (14) and seeded at a rate of $10^{5}$ cells per well in 96-well plates. Thereafter, the proportion of parasitized RBCs was adjusted by dilution so as to distribute in a volume of $200 \mu \mathrm{l}$ a statistical $0.3-0.5$ parasites (further checked by IFA as described above). In addition, a well containing 1,000 infected RBCs per well was prepared so as to obtain a fast estimate of the quality of growth. Every $8 \mathrm{~d}$, fresh feeder cells were prepared, and the content of each well was resuspended using a multichannel eight-tip pipette and transferred onto fresh hepatocytes. Culture medium consisted of RPMI 1640 supplemented with insulin, albumin (fraction V; Sigma Chemical Co., St. Louis, MO), corticosteroids as described (14), and 10\% human fetal serum from umbilical cord (19). No double cloning was attempted in order to reduce to a minimum the time the parasite spent in culture before characterization.

The remainder of the cloning procedure was done essentially as described above (Fast cloning procedure). Whenever positive wells were detected, the cell suspension was transferred to 24-well plates 
and thereafter to $35-\mathrm{mm}$ petri dishes, both seeded with hepatocyte feeder layers. Starting from day 42 , attempts were made at regular intervals to adapt positive clones to standard culture conditions, without adding feeder layers. Thereafter, some aliquots were cryopreserved, while others were used to produce a large number of parasites in 100 -mm petri dishes.

In all, 31 isolates (20 drug-sensitive and 11 drug-resistant; 23 cloned in Paris, 8 in Bangkok) were included in the above process.

Assessment of drug sensitivity. For all isolates and clones, the response towards chloroquine, quinine, and mefloquine was studied using an isotopic technique described previously (20). A range of 11 concentrations was prepared for each drug from extemporaneously made solutions $(0.48-500 \mathrm{ng} / \mathrm{ml}$ for chloroquine and quinine, 0.24 $250 \mathrm{ng} / \mathrm{ml}$ for mefloquine). All assays included as internal controls the Uganda Palo Alto isolate and the 3D7 clone, whose sensitivity had been evaluated regularly and was determined simultaneously. Results are expressed as the concentration of drug inhibiting the hypoxanthine uptake by $50 \%\left(\mathrm{IC}_{50}\right)$. Variations in the $\mathrm{IC}_{50}$ of these reference strains in the successive assays never exceed $10-15 \%$. The cut-off drug concentration between sensitivity and resistance to each drug had been determined previously by comparative in vivo/in vitro drug sensitivity assays (21).

Parasite DNA preparation. DNA from isolates and cultured clones was extracted and purified as described previously (22) except that DNA used for PCR was not dialyzed.

For RFLP analysis, $4 \mu \mathrm{g}$ of genomic DNA was digested to completion using an excess of restriction enzymes. For karyotype analysis, all parasites were grown to $\sim 10 \%$ parasitemia, and chromosomal blocks were prepared according to Van der Ploeg et al. (23).

PCR conditions. Amplification was performed on clone or isolate DNA extracted from $\sim 5 \times 10^{7}$ RBCs in a final volume of $50 \mu \mathrm{l}$ (using $2 \mathrm{U}$ Taq polymerase, $1 \times$ buffer [Amersham Corp., Arlington Heights, IL], $0.2 \mathrm{mM}$ deoxynucleotide-triphosphate, and $50 \mathrm{pmol}$ of each primer). The sequence of the primers used (described in references 10 and 24), was as follows: (a) MSP-1, (+)5'-AAGCTTTAGAAGATGCAGTATTGAC-3' and (-)5'-ATTCATTAATTTCTTCATATCCATC-3'; MSP-2, (+)5'-AACGAATTCATAAACAATGCTTATAATATGAGT- $3^{\prime}$ and $(-) 5^{\prime}$-GATGAATTCTAGAACCATGCATATGTCCATYGTT-3'; thrombospondin-related anonymous protein (TRAP), (+)5'-ATGTAACTTGTATGCTGATTCTGCATGG-3' and (-)5'-TATCTTCACTATTAGGTACGTGCCTATTTCC-3'; and circumsporozoite (CS), (+)5'-AGAGATGGAAATAACGAAGACAACGAG-3' and (-) 5'-GTCATTTGGCATTAAGTGACCTTGTCC-3'.

Incubation conditions were as follows: $94^{\circ} \mathrm{C}$ for $2 \mathrm{~min}, 55^{\circ} \mathrm{C}$ for 1 $\mathrm{min}$, and $72^{\circ} \mathrm{C}$ for $1 \mathrm{~min}$, followed by 35 cycles at $94^{\circ} \mathrm{C}$ for $4 \mathrm{~s}, 55^{\circ} \mathrm{C}$ for $30 \mathrm{~s}$, and $72^{\circ} \mathrm{C}$ for $30 \mathrm{~s}$. The amplification was completed by $5 \mathrm{~min}$ incubation at $72^{\circ} \mathrm{C} .10 \mu \mathrm{l}$ of the PCR reaction was analyzed as described below.

Electrophoresis and DNA transfer conditions. For RFLP analysis, digested DNA was loaded on a $0.8 \%$ agarose gel and run overnight in $1 \times$ TBE buffer ( $89 \mathrm{mM}$ Tris, $89 \mathrm{mM}$ boric acid, $2 \mathrm{mM}$ EDTA) at 35-40 V.

For pulsed field gradient electrophoresis (PFGE), chromosomal blocks were sealed in $1 \%$ agarose gels and run in $0.5 \times \mathrm{TBE}$ buffer as described previously (25). Gels were then stained in $0.5 \mu \mathrm{g} / \mathrm{ml}$ ethidium bromide at room temperature for $1 \mathrm{~h}$, destained in distilled water for several hours, and photographed under a standard ultraviolet (UV) transilluminator.

$10 \mu \mathrm{l}$ of PCR reaction products was loaded on $3 \%$ NuSieve $3: 1$ agarose (FMC Bioproducts, Rockland, ME) gels containing $0.5 \mu \mathrm{g} / \mathrm{ml}$ ethidium bromide, run 40-60 min in $1 \times \mathrm{TBE}$ buffer at $80 \mathrm{~V}$, and photographed under a standard UV transilluminator. RFLP and PCR gels were processed and blotted to nylon membranes (Hybond-N; Amersham Corp.) according to standard protocol (26).

Probes, labeling, and hybridization conditions. The repetitive rep20 probe was isolated from a HindIII/EcoRI genomic library constructed in pUC9. Cloning and sequencing are described elsewhere
(25). MSP-1 and MSP-2 probes are described elsewhere in detail (24). Briefly, MSP-1 probes correspond to amplification products of block 2 sequences, specific for Camp, Wellcome, and RO33 alleles (27, 28); MSP-2 probes correspond to amplification products of sequences specific for IND/3D7 and FC27 alleles (29).

Probes were ${ }^{32} \mathrm{P}$-labeled using a random priming kit (Amersham Corp.). Hybridizations were carried out at $65^{\circ} \mathrm{C}$ in $6 \times \mathrm{SSC}(0.9 \mathrm{M}$ $\mathrm{NaCl}, 0.09 \mathrm{M}$ sodium citrate), $5 \times$ Denhardt's solution, $0.5 \% \mathrm{SDS}$ (wt/ vol). Washes were done at $65^{\circ} \mathrm{C}$ in $2 \times \mathrm{SSC} / 0.1 \%$ SDS followed by $0.5 \times \mathrm{SSC} / 0.1 \%$ SDS (for PCR blots) or $0.1 \times \mathrm{SSC} / 0.1 \% \mathrm{SDS}$ (for RFLP blots) before autoradiography.

\section{Results}

Cloning P. falciparum fresh isolates. Preliminary studies aimed at optimizing the variable hematocrit cloning procedure were conducted in 24-well plates using the NF54 and FUP/C strains, adapted previously to culture conditions, from which 8 and 12 clones were derived, respectively. Thereafter, cloning in 96-well plates from freshly collected isolates was attempted. 15 isolates of African origin were cloned successfully in Paris, 4 of Thai origin in Bangkok. The simultaneous cultivation of the uncloned isolates with and without feeder hepatocytes confirmed our earlier observations: when using feeder cells, the speed of growth and reinvasion of new RBCs was close to optimal (2-5 times in $48 \mathrm{~h}$ ), whereas without feeders, we observed, as have many others, that one or two cycles of maturation occurred, but the reinvasion rate was so low that the parasitemia was undetectable in all studied isolates by day 15 . A typical example is given in Fig. 1.

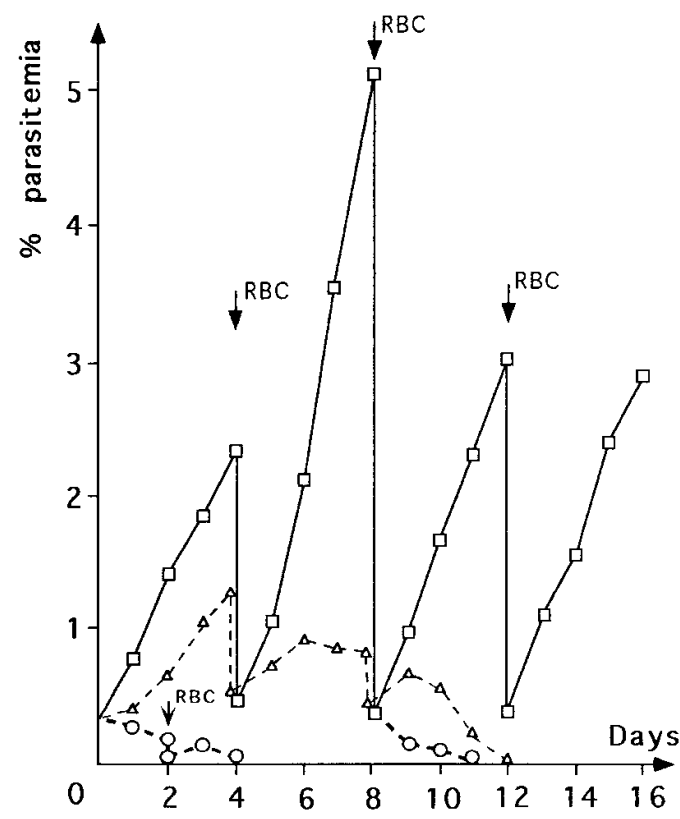

Figure 1. Improved growth of the P. falciparum African isolate when cultured with hepatocyte feeder cells (squares, solid line), compared with culture without feeder cells (circles), or with hepatocyte culture supernatants (triangles). This culture of the uncloned isolate was performed in parallel with the cloning procedure, also starting on the day of sampling (day 0) but in 24-well plates. Arrows, Time of subcultures. On day 8 , a second attempt of isolate cultivation without feeders or hepatocyte supernatant was performed, without success. 
The cloning procedure proved both fast and efficient, requiring $\sim 1 \mathrm{~h}$ work every $3 \mathrm{~d}$ and $4-5 \mathrm{~h}$ on days 15,21 , and 27 . However, not all clones produced could be kept on feeders and further expanded in standard conditions, i.e., without feeders. For example, 75 clones obtained from 192 wells seeded with one Cameroonese isolate were lost after $5 \mathrm{wk}$ of cultivation. For further studies, we kept two series of 34 and 33 clones derived from one Ivory Coast (101) and one Thai (TB2) isolate, respectively (cloned in 96 wells). Note that the output of cloning (33 and 34/96, 75/192) was close to the maximal theoretical output of $37 \%(30)$, which is higher than reported for previous clonings (30). From these results, it can be concluded that most of the clones produced under these conditions would not have been isolated in standard culture conditions.

Pattern of response to three drugs. Studies performed with each individual clone revealed a large diversity amongst the clones derived from each isolate in terms of their sensitivity to antimalarial drugs (Fig. 2). Dose-response curves were very regular over the range of concentrations tested, leaving little doubt as to the $\mathrm{IC}_{50}$ determined. In addition, 12 African and 6 Thai clones were tested twice and yielded almost identical results. Even accepting a $10-15 \%$ error, the range in $\mathrm{IC}_{50}$ is important, and the diversity is striking. Furthermore, the combined patterns given by the individual responses to each of the three drugs studied were almost all unique, providing the first strong suggestion of wide phenotypic differences between individual parasites constituting each isolate.

The range of responses of the clones from the Thai isolate to chloroquine and quinine (45-240 and 47-200 ng/ml, respectively), or of the clones from the African isolate to quinine (14.5-107.5 $\mathrm{ng} / \mathrm{ml})$, was much wider than that obtained in clones from NF54 and FUP/C lines which had already been adapted to culture conditions (chloroquine: NF54, 7.8-11.5 $\mathrm{ng} / \mathrm{ml}$, and FUP/C, 61-96 ng/ml; quinine: NF54, 28-52 ng/ml, and FUP/C, 44-56 ng/ml [data not shown]). These results indicate that under the culture conditions used, clones of greater diversity were retained. In agreement with this observation, the drug sensitivity of the uncloned Thai isolate (first adapted using feeder cells, then cultured without) increased from 45 to $150 \mathrm{ng} / \mathrm{ml}$ for chloroquine and from 30 to $130 \mathrm{ng} / \mathrm{ml}$ for quinine after $70 \mathrm{~d}$ of culture (whereas the sensitivity of control lines NF54 and Palo Alto remained unchanged). In other words, in the uncloned line, only the drug-resistant clones predominated, or survived at the end of this culture period.

Both chloroquine- and quinine-resistant clones were found in the Thai isolate, whereas all clones from the Ivory Coast isolate were sensitive to chloroquine, in agreement with the status of response to these drugs in the two countries at the time of sampling (1985). In contrast, one quinine-resistant and four mefloquine-resistant clones were derived from the overall sen-
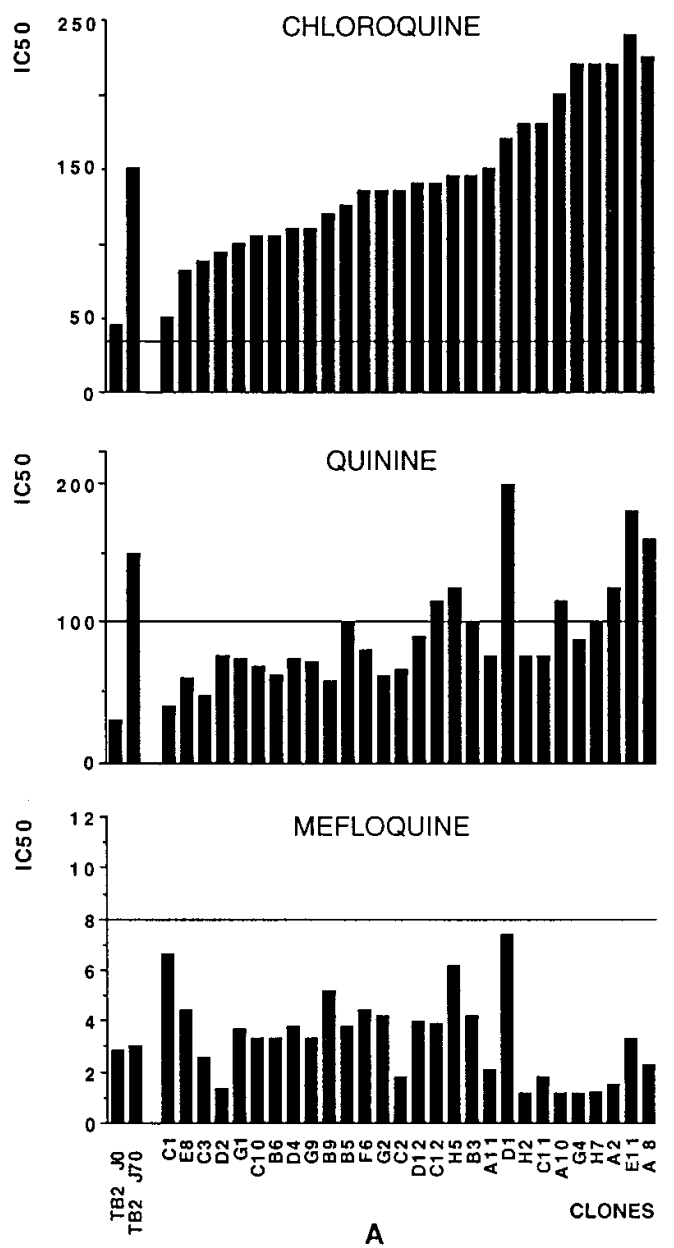
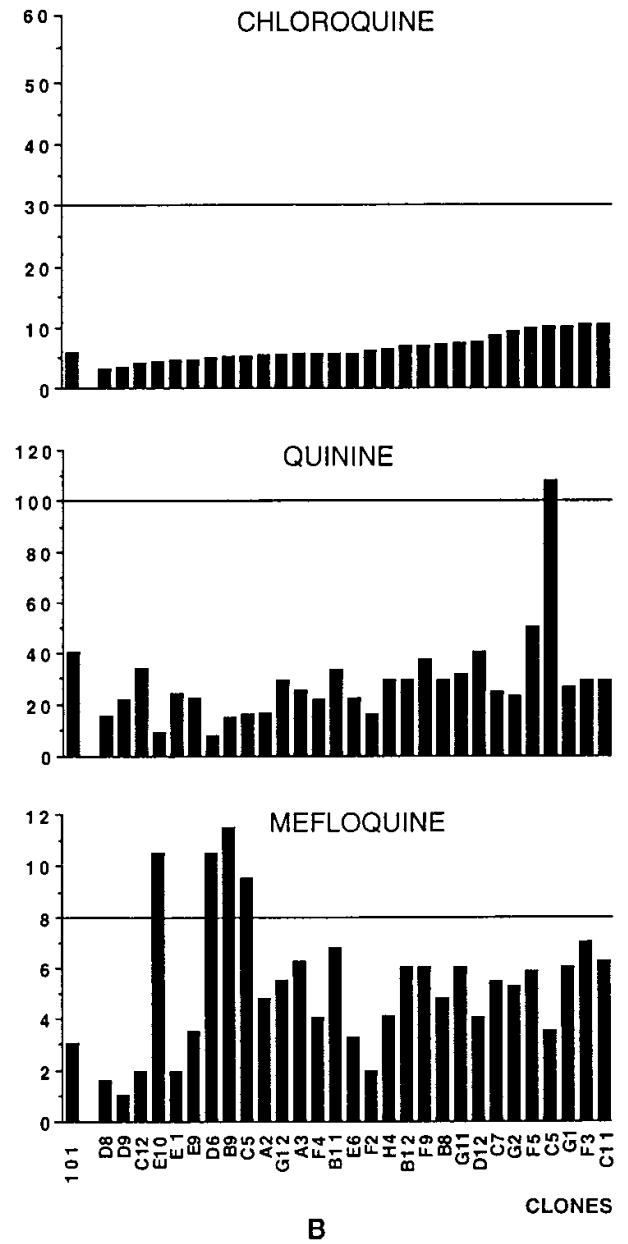

Figure 2. Complex pattern of responses to three drugs obtained with the Thai and the African clones indicates wide phenotypic differences among the various clones derived from each isolate. (A) Thai isolate and clones: TB2 J0, TB2 isolate on the day of sampling; TB2 J70, TB2 isolate after $70 \mathrm{~d}$ cultivation; and TB2-derived clones. (B) African isolate and derived clones. The $\mathrm{IC}_{50}$ are expressed in nanograms per milliliter; note the scale variation depending on the drug and the isolate studied. Horizontal bars, Cut-off values between sensitivity and resistance (31). Clones are classified in order of increasing resistance to chloroquine, and the corresponding sensitivity to quinine and mefloquine is shown below for each. 
sitive isolate from the Ivory Coast (Fig. 2). No clear pattern of cross-resistance between any of the three drugs was seen in the African clones (see, for example, clones C1, D12, C12, H5, and B3 in Fig. 2). In contrast, some of the Thai clones (e.g., clones A11, E2, and E11 in Fig. 2) showed a cross-resistance between chloroquine and quinine $(r=0.66)$, confirming what had been observed in the field, that chloroquine resistance is accompanied by quinine resistance in some locations though not in others (31). Finally, two quinine-as well as mefloquine-resistant clones were also derived from a Cameroonese isolate ( $\mathrm{IC}_{50}$ values: mefloquine, 30 and $45 \mathrm{ng} / \mathrm{ml}$; quinine, 96 and 100 $\mathrm{ng} / \mathrm{ml}$ [data not shown]), even though at the time we sampled our isolates (1985), no mefloquine resistance had been reported in Cameroon. However, 3 yr later, many mefloquineresistant isolates had emerged $(20,32)$. In the case of the Cameroon isolate, the cross-resistance between mefloquine and quinine found in our clones was similar to that reported for isolates studied later in the field (31). This is consistent with the hypothesis that quinine pressure is responsible for the emergence of isolates resistant to mefloquine (Lariam) $(31,33)$.

Karyotype analysis. PFGE was applied to nine of the Thai and eight of the African clones. Shown in Fig. 3 are profiles obtained under conditions which better demonstrate the variation in size of the smaller chromosomes. This was sufficient to differentiate each of the eight African clones and eight of nine of the Thai clones.

PFGE analysis of different cultured isolates is known to yield discrete chromosomes $(5,23,34)$. However, the unique karyotype profile of an isolate is surprising in view of the wide range of chromosome sizes in our clones. To evaluate the sensitivity of the PFGE technique, parasites from Thai clones G9 and C11 were mixed artificially in equal amounts and submitted to PFGE separation. Their individual karyotypes could be readily observed in this reconstituted simple mixture (Fig. 3). Therefore, PFGE can resolve mixed isolates, providing the composite clones are present in relatively equal proportions. Thus, a unique karyotype of a cultured isolate implies that a predominant clone is selected under standard culture conditions, a view consistent with the indications given by our drug studies (see above).

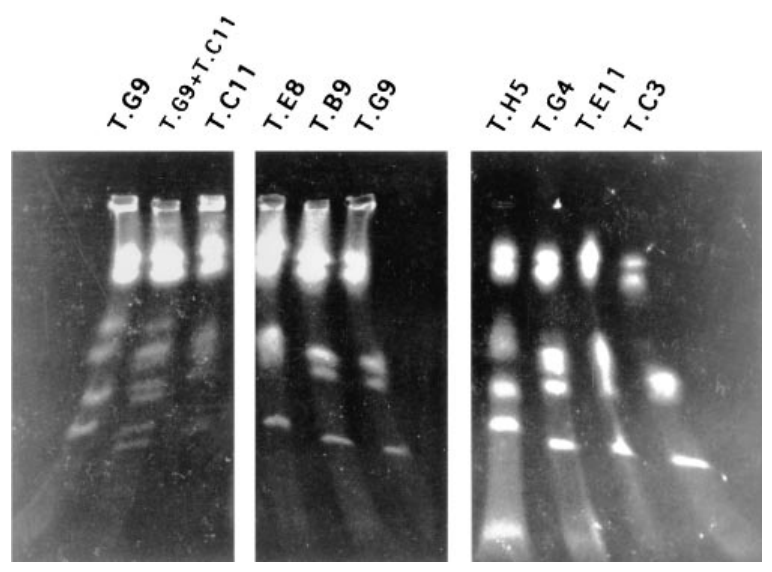

Figure 3. PFGE separation of just the smaller chromosomes led to the differentiation of eight of nine Thai clones. T.G9+ T.C11, An equimolar mixture of the two clones, demonstrating that PFGE can resolve an artificially mixed simple isolate.
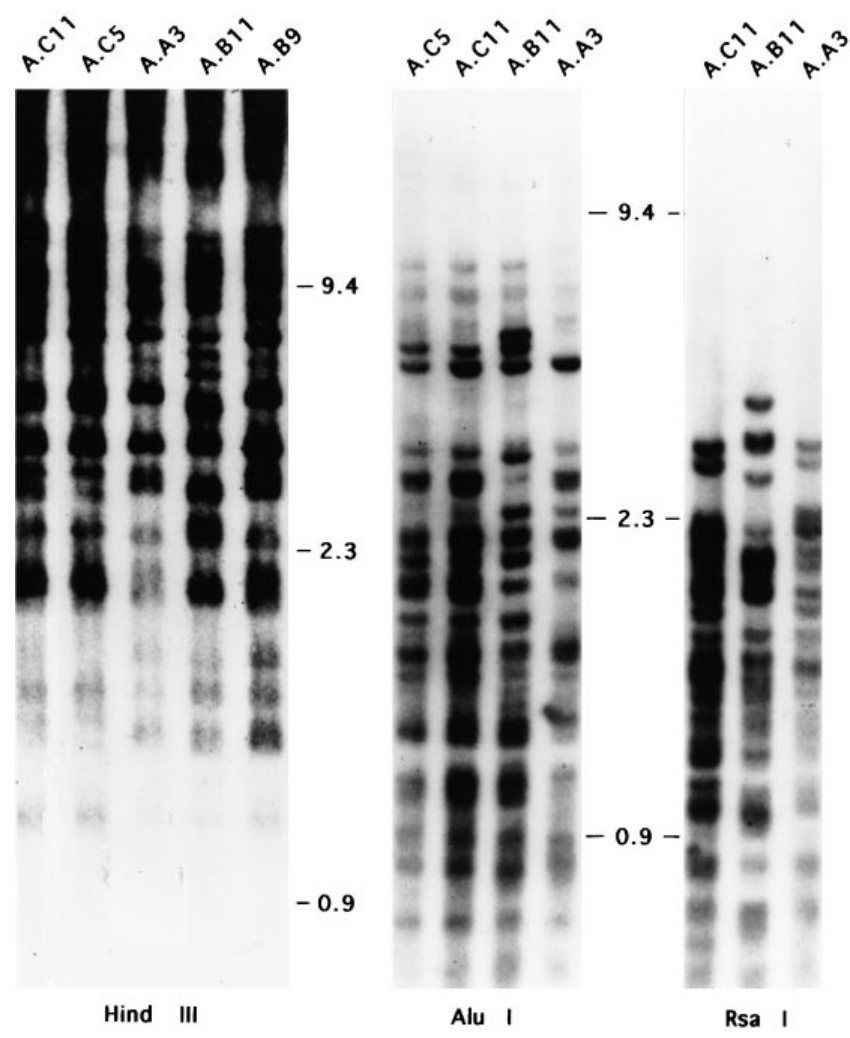

Figure 4. The RFLP patterns obtained with the DNA from African clones after HindIII, AluI, and RsaI digestions followed by rep 20 probe hybridization revealed restriction site differences (but also similarities of pattern) between each of the studied clones from this isolate. Molecular weight markers are expressed in kilobases.

RFLP patterns. RFLP analysis is an efficient and widely used tool for malaria strain typing (35-37). Although polymorphic single gene probes such as the $S$ antigen were used (data not shown), the highly repetitive rep20 probe proved more discriminatory. As expected, restriction enzymes with A+T-rich recognition sites, such as AluI, HindIII, RsaI, and TaqI, gave more complex and informative patterns. Among the two sets of clones from the African (Fig. 4) and Thai (Fig. $5 \mathrm{~A}$ ) isolates, digestion by a single enzyme produced rep20 RFLP patterns which clearly differentiated each of the individual clones studied by one or several bands. Patterns given by additional enzymes simply confirmed this observation (Fig. 4, and data not shown). The RFLP differences in the rep20 patterns were probably not due to deletions of rep20 during cultivation of the parasites, since the patterns of individual clones remained unchanged after cultivation, i.e., in the case of clone $\mathrm{C} 11$, for 4 mo of continuous culture (Fig. $5 \mathrm{C}$ ). Importantly, although rep20 revealed differences between clones of a single isolate, the fingerprints of individual clones from a single isolate showed clear similarities. This contrasts with the numerous differences in RFLP patterns between unrelated strains (35). Shown as an example is the RFLP pattern obtained from two independent isolates (Fig. $5 \mathrm{~B}$ ). This suggests a degree of genetic relatedness among the clones of a given isolate which was not predicted by karyotype profiles.

PCR analysis. Heterogeneity of the clones was further assessed using PCR with DNA from 27 Thai and 27 African 


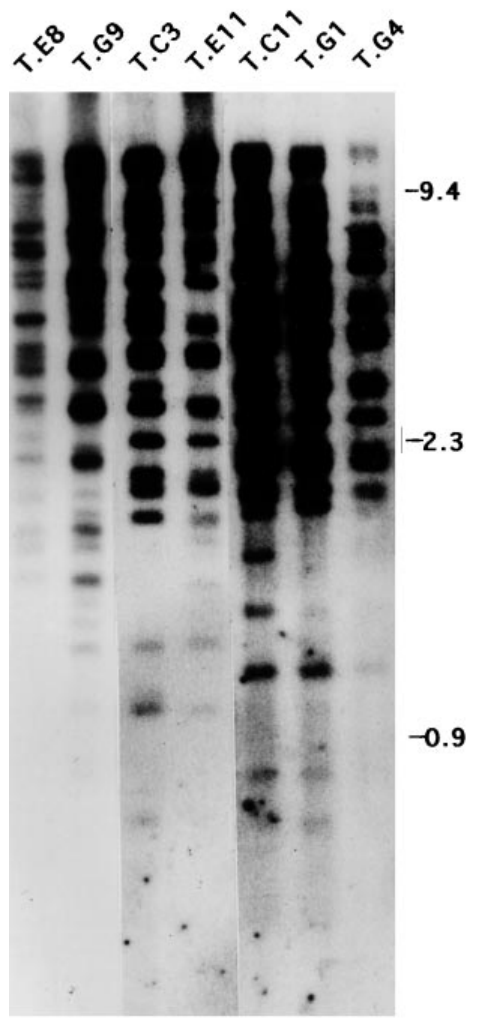

A

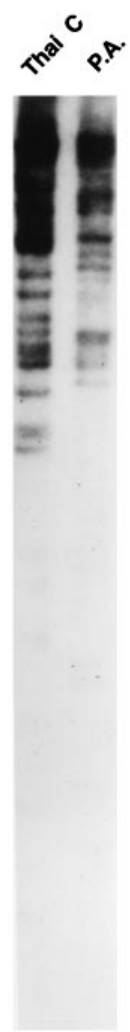

B
T.C11

a b

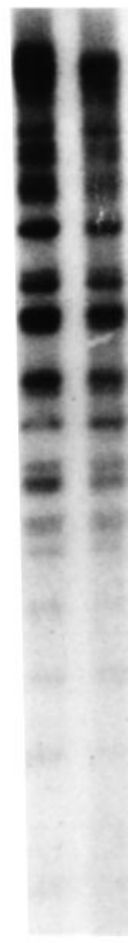

C

Figure 5. (A) Examples of HindIII RFLP patterns of seven Thai clones after digestion and rep20 hybridization, which distinguishes all clones from one another. $(B)$ HindIII RFLP patterns of an independent Thai sample (Thai $C$ ) isolated at the same time from the same geographical region, together with the Palo Alto (Uganda) (P.A.) strain. (C) RFLP patterns of T.C11 clone before $(a)$ and after $(b) 4$ mo cultivation. Molecular weight markers are expressed in kilobases.

clones. For each clone, variable regions of the four polymorphic genes coding for MSP-1, MSP-2, TRAP, and the CS (for a review, see reference 38) were amplified according to a procedure described in greater detail elsewhere $(11,24)$. The specificity of the amplification was confirmed by Southern blotting and hybridization with probes specific for each polymorphic marker (data not shown).

Analysis of the polymorphic loci of the clones of both isolates revealed a specific pattern of amplification which was determined for each clone by combination of the four markers (Fig. 6). The combination of the PCR patterns of the four alleles (Table II) allowed the classification of the clones into groups. A single group predominated $(67 \%)$ in each isolate. Detection of the same PCR band in a number of clones was expected, given the genetic relatedness between the clones demonstrated above by rep20 fingerprinting. Among the series of clones making up the two isolates, three African and three Thai clones revealed two bands with at least one marker (*) and are probably clonal mixtures. This is likely due to the presence in the original isolates of erythrocytes containing more than one parasite which were not eliminated by the single round of dilution cloning. PCR analysis was also performed on whole isolate DNA prepared at the time of sampling, i.e., before cloning, and again after $>10$ mo of cultivation of the isolate. A complex PCR pattern was observed corresponding to the sum of the amplification products obtained with the individual clone derived from the corresponding isolate. No additional band was detected in the isolates. This provided an indication of the efficiency of the cloning procedure, since all the subpopulations detected in the isolates were indeed identified also in the two series of clones. Furthermore, it indicates that no profound genetic modification within the polymorphic regions of the markers was generated during cloning of the isolates and their subsequent cultivation.

Comparative analysis of the results supplied by distinct markers. A combined analysis of the results yielded by the

Table II. Combination of PCR Patterns

\begin{tabular}{|c|c|c|c|c|c|c|c|}
\hline Isolate & MSP-1 & MSP-2 & CSP & TRAP & Pattern & Total & Clones \\
\hline & 2 & 1 & 2 & 1 & A & $19 / 27$ & $\begin{array}{l}\mathrm{A} 2, \mathrm{~A} 8, \mathrm{~A} 10, \mathrm{~B} 5, \mathrm{~B} 6, \mathrm{~B} 9 \mathrm{C} 2, \mathrm{C} 3, \mathrm{C} 11, \mathrm{C} 12^{*}, \\
\mathrm{D} 2, \mathrm{D} 4, \mathrm{D} 12^{*}, \mathrm{E} 2, \mathrm{E} 8, \mathrm{E} 11, \mathrm{G} 1, \mathrm{G} 4, \mathrm{H} 2\end{array}$ \\
\hline \multirow[t]{5}{*}{ TB2 } & 1 & 2 & 1 & 2 & $\mathrm{~B}$ & $8 / 27$ & $\mathrm{~A} 11, \mathrm{C} 10, \mathrm{C} 12 *, \mathrm{D} 1, \mathrm{D} 12^{*}, \mathrm{~F} 6, \mathrm{G} 9, \mathrm{H} 5$ \\
\hline & 2 & 1 & 2 & 3 & $\mathrm{C}$ & $2 / 27$ & $\mathrm{G} 2, \mathrm{H} 7$ \\
\hline & - & - & - & - & Mixed & $2 / 27$ & $\mathrm{C} 12 *, \mathrm{D} 12 *$ \\
\hline & 1 & 2 & 1 & 3 & A & $4 / 27$ & $\mathrm{C} 5, \mathrm{C} 12 *, \mathrm{D} 6, \mathrm{E} 6$ \\
\hline & 1 & 2 & 2 & 1 & $\mathrm{~B}$ & $18 / 27$ & $\begin{array}{l}\text { A2, B8, B9, B12*, C7, C11, D8, D12, } \\
\text { E1, E10, F3, F5, F9, G1, G4, G12, H4 }\end{array}$ \\
\hline \multirow[t]{5}{*}{101} & 1 & 3 & 2 & 1 & $\mathrm{C}$ & $2 / 27$ & $\mathrm{~B} 11, \mathrm{~B} 12 *$ \\
\hline & 2 & 1 & 2 & 2 & $\mathrm{D}$ & $1 / 27$ & A3 \\
\hline & 2 & 2 & 1 & 1 & $\mathrm{E}$ & $1 / 27$ & E9 \\
\hline & 2 & 3 & 1 & 3 & $\mathrm{~F}$ & $3 / 27$ & $\mathrm{C} 12^{*}, \mathrm{~F} 2, \mathrm{~F} 4$ \\
\hline & - & - & - & - & Mixed & $2 / 27$ & $\mathrm{~B} 12 *, \mathrm{C} 12 *$ \\
\hline
\end{tabular}

PCR analysis of four polymorphic loci (MSP-1, MSP-2, CS [CSP], and TRAP) on clones from the Thai isolate (TB2) and the African isolate (101). Numbers $(1,2$, or 3$)$ correspond to a specific band size for each locus, and when summed for the four loci give rise to a particular pattern, indicated as $A, B, C, D, E$, and $F$. The different clones presenting each pattern are given (Clones) as well as the number of clones of each isolate with a particular pattern (Total). Mixed, Possible clonal mixtures. Note that for both isolates, 2/3 of the clones group into a single pattern. *Clonal mixtures. 


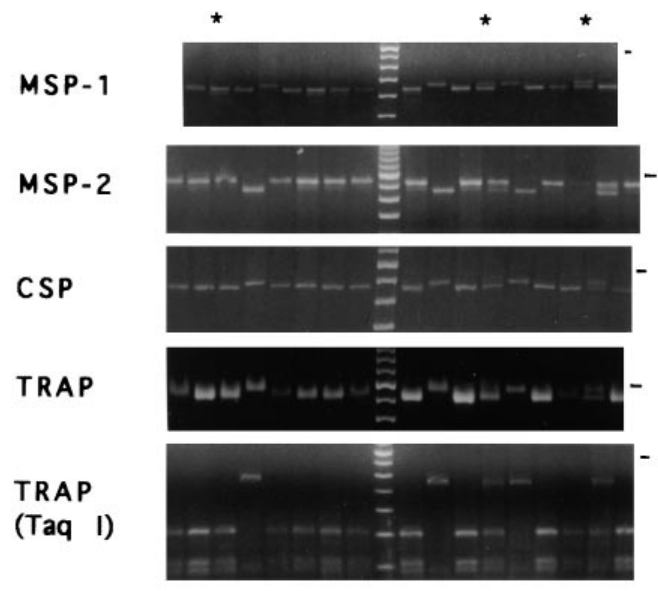

A

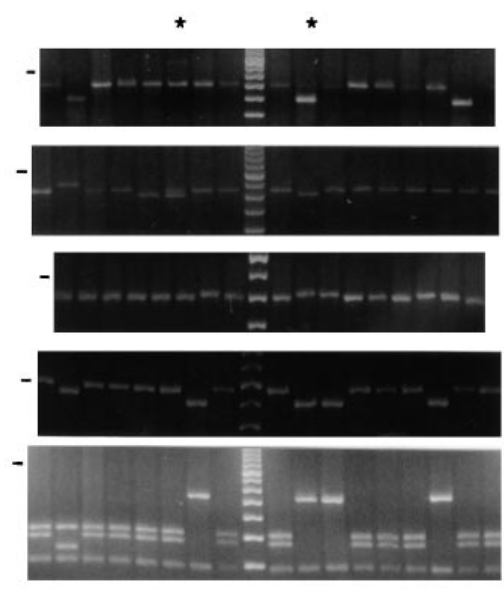

B
Figure 6. Genotypic differences among clones were also demonstrated by PCR amplification of polymorphic loci. Shown are examples with DNA from $(A)$ the Thai and $(B)$ the African clones. (C) PCR amplification of the MSP-1 marker with the Thai isolate (far left), the Thai isolate after 6 mo cultivation (middle left), the African isolate (middle right), and the African isolate after 6 mo cultivation (right). *Clonal mixtures. Molecular weight markers are 100-bp ladders from GIBCO BRL. The 800-bp band is shown with a dash. CSP, CS. nine different markers on the two isolates amply illustrates the degree of diversity, while also demonstrating the genetic relatedness between clones. Fig. 7 shows the analysis of six clones indistinguishable by PCR and their comparison to a markedly different clone (G9) of the same Thai isolate. Allelic size polymorphism estimated by PCR clearly underestimates diversity, since all six clones demonstrated karyotype variation, RFLP differences (Fig. 5), and distinct patterns of drug response (Fig. 2). For example, clone $\mathrm{C} 3$ is three times more susceptible to either chloroquine or quinine than clone $\mathrm{E} 11$ (see $\mathrm{IC}_{50}$ in Fig. 2). Nonetheless, their RFLP fingerprints are strikingly similar, showing only a limited number of clear-cut differences in the number of bands (see Fig. 5). Thus, a single inoculum is composed of genetically related parasites (implying the same parents), yet recombination is sufficient to generate diversity. Similar analysis of the clones derived from the African isolate

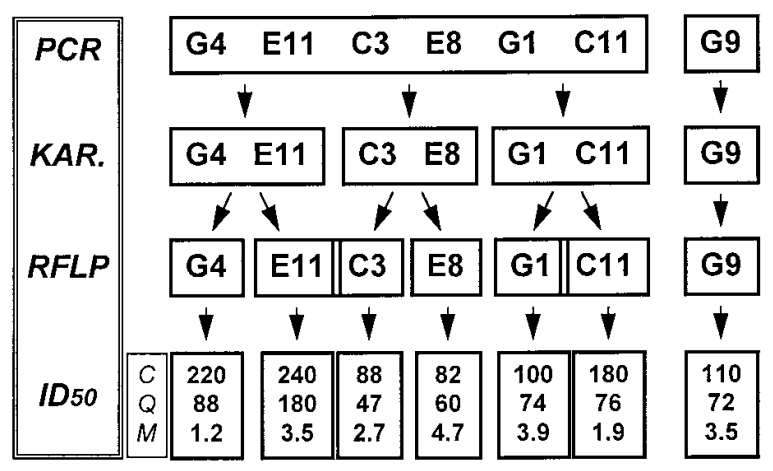

Figure 7. Comparison of phenotypic markers for six clones showing an identical PCR pattern, and their comparison with a different clone of the same Thai isolate. Boxed, Indistinguishable phenotype. $K A R$, Karyotype profile. $R F L P$, rep20 fingerprint. For $\mathrm{IC}_{50}$, in the same box is shown the $50 \%$ inhibitory dose $\left(I D_{50}\right)$ for chloroquine $(C)$, quinine $(Q)$, and mefloquine $(M)$. Note that even very closely related clones (E11 and C3) differ in their drug responses. was performed and yielded the same conclusions (data not shown).

\section{Discussion}

Our study suggests that the complexity of malaria isolates, although documented previously, has been significantly underestimated, and that previous PCR typing of field isolates yielded a suboptimal estimate of parasite diversity. Moreover, it has been generally assumed that isolate diversity results from superinfection, i.e., is the sum of successive inoculations in exposed individuals (1-4), but the contribution of a single mosquito bite has not been clearly examined. To address this question, we have searched for primary isolates, where due to short term exposure or low endemicity, the probability that the infection arises from a single mosquito bite was extremely high. Furthermore, to avoid introducing a bias into our analysis, we developed a cloning culture process which was initiated on the day of sampling, before in vitro growth of the isolate, thereby avoiding selective pressure on the clones. In this way, a total of 33 and 34 clones were obtained from a Thai and an African isolate, respectively. This corresponds to a cloning yield of $35 \%$, which is close to the maximal theoretical rate of $37 \%$ (30). The clones were then characterized by PCR, RFLP, and chromosome analysis, as well as by their response to varying doses of three different drugs.

Two important findings emerge from this study. First, the clones of a given isolate appeared to have a common genetic background, since they displayed closely related RFLP patterns. This relatedness is evident even though subtelomeric instability (potentially affecting rep20 RFLP patterns) ought to exaggerate any apparent diversity. Furthermore, the RFLP patterns of these clones are characteristic for each isolate, whereas different isolates from the same region have markedly altered fingerprints (Fig. $5 \mathrm{~B}$ ). In agreement with this finding and consistent with the assumption that the cloned parasites are derived from a single inoculum, $\sim 2 / 3$ of the clones presented the same combination of four PCR markers known to be genetically independent (Table II). 
Second and more important, in spite of this genetic relatedness, clones from the same isolate exhibit an unexpectedly high degree of diversity (Fig. 7). Indeed, clones that were indistinguishable by PCR (e.g., C3 and E11) showed clear-cut differences in all other markers, e.g., karyotype or fingerprints, and showed significant variation in their responses to the three antimalarial drugs tested. In total, this cannot be explained by variability in the different biological assays and is thus highly significant. This suggests that a previously unappreciated amount of genetic diversity is likely to exist in a single isolate. Incidentally, it should be emphasized that our study demonstrates that PCR typing strongly underestimates isolate diversity. This could be expected, given the limited fraction of the parasite genome examined by the set of primers currently used.

The relatedness of the individual clones implies that they were generated by genetic recombination between a limited number (likely only two) parents. Under field conditions, it has been reported that infected mosquitoes harbor most frequently one and only sometimes two oocysts, the progeny of only two and occasionally four gametocytes (39, 40). Even though RFLP and PCR typing of the clones cannot prove that they are siblings, our data are consistent with this observation. Indeed, 90\% (27/29) and 86\% (25/29) of the Thai and the African clones, respectively, contain no more than two alleles at each of the four polymorphic loci examined by PCR. Furthermore, $100 \%(27 / 27)$ and $88 \%(22 / 25)$ of these clones present only two combinations of these four independent markers. Therefore, for both isolates, PCR patterns A and B (Table II) are most likely explained as a combination of the two parental allelic patterns. The main conclusion is that meiotic recombination is likely to be the major generator of diversity in the two isolates we studied, although the extent to which liver and blood schizogony may contribute to this remains to be explored.

Is genetic recombination in malaria parasites high enough to generate the observed diversity? Two pieces of evidence suggest that it might be. First, in laboratory-infected mosquitoes, PCR analysis of the MSP-1 locus showed that $50 \%$ of oocysts were hybrid (41). Second, in an experimental genetic cross, the recombination frequency was always higher than expected: only 3 of 22 progeny clones expressed the parental type $(42,43)$. Our study now suggests that this diversity also exists in natural populations.

Diversity arising from the sexual phase of replication in the mosquito would provide the parasite with the greatest potential for survival, with some clones better fit to adapt to a given MHC type, a given RBC phenotype, or to face a given innate or acquired immune response, that is to "adapt" to various situations either in vitro or in vivo in humans or in primates in experimental conditions.

The combined responses to three drugs extend previous observations in this field $(4,44)$ by showing $(a)$ a wide diversity in the susceptibility of individual clones to each drug, and moreover, $(b)$ a very diverse range of individual sensitivity patterns of each clone to each of the three drugs tested. When culturing the uncloned isolates, drug sensitivity to both quinine and chloroquine dropped dramatically and rapidly after cultivation, reflecting the emergence of the most drug-resistant clones. This result indicates that the drug sensitivity shift described previously in isolates (6) was most likely due to a strong selective process exerted by culture conditions in favor of the resistant parasites subset. This also stresses the advantage of using a cloning procedure that avoids any prior cultivation of the isolate.

Drug resistance is undoubtedly today's greatest threat to malaria control. In this respect, the complex pattern of responses observed in each clone provides new and important information. The wide diversity of drug response phenotypes is in favor of the notion that several loci are involved in the resistance to each drug and that the loci segregate independently. For example, the phenomenon of cross-resistance between chemically related drugs, e.g., chloroquine and quinine, or quinine and mefloquine, which has been reported previously in field isolates $(31,45)$, is also observed here at the individual clone level but is not seen in all resistant clones. This clearly indicates that several individual genes, not a single one, can be involved in resistance to a given compound. Finally, the patterns obtained also show that these resistance genes are not linked but segregate independently.

A demonstrative example of the strategic advantage given to a parasite isolate by its clonal diversity is supplied by the results we obtained with mefloquine. Interestingly, four of the clones from the Ivory Coast were resistant to mefloquine. In addition, three clones of 75 produced from a Cameroonese isolate (192-well cloning, data not shown) also appeared to be resistant to mefloquine $\left(\mathrm{IC}_{50}>10 \mathrm{ng} / \mathrm{ml}\right)$, two of which though not the third were also resistant to quinine. Although at the time of sampling (1985), no cases of resistance to this drug had been reported (in agreement with the overall sensitivity of the two above uncloned isolates), mefloquine resistance indeed emerged 3 yr later in Cameroon (20) and increased in frequency and level of resistance in the following year $(31,32)$. Thus, the results suggest that these particular parasites were already circulating and only required the clinical use of mefloquine, or more likely the structurally related drug quinine (which is widely used in French-speaking countries of Africa), to be selected. Among the various hypotheses proposed (21) to explain the sudden emergence and subsequent spread of drug-resistant Plasmodia in Africa, the present results reinforce the notion of clonal selection (of a quantitatively minor resistant parasite population preexisting among large numbers of sensitive clones) under drug pressure.

In conclusion, although the cultivation procedure we used is more fastidious than standard techniques, it permitted the production, maintenance, and analysis of parasite clones that otherwise would have been lost. This analysis revealed a level of clonal complexity in primary isolates which had not been foreseen, but given the experimental set-up, are hardly explained by multiple infections. Injection by mosquitoes of phenotypically diverse inocula not only assures successful survival of viable parasites in genetically diverse hosts, but also is a potential threat for drug treatment, vaccine development, and malaria control in general.

\section{Acknowledgments}

We are grateful to all staff members of the Department of Tropical Pediatrics, Mahidol University, for their assistance, and to David Fidock, Dominic Kwiatowski, Odile Mercereau-Puijalon, Louis Miller, Piero Olliaro, Richard Paul, and David Walliker for useful discussions.

This work was supported by European Economic Community grant CT910884 and World Health Organization Tropical Diseases Research Fieldmal grant 840111. 


\section{References}

1. Daubersies, P., S. Sallenave-Sales, S. Magne, J.-F. Trape, H. Contamin, T. Fandeur, C. Rogier, O. Mercereau-Puijalon, and P. Druilhe. 1996. Rapid turnover of Plasmodium falciparum populations in asymptomatic individuals living in a high transmission area. Am. J. Trop. Med. Hyg. 54:18-26.

2. Scherf, A., D. Mattei, and J.L. Sarthou. 1991. Multiple infections and unusual distribution of block 2 of the MSA1 gene of Plasmodium falciparum detected in West African clinical isolates by polymerase chain reaction analysis. Mol. Biochem. Parasitol. 44:297-299.

3. Snewin, V.A., M. Herrera, G. Sanchez, A. Scherf, G. Langsley, and S. Herrera. 1991. Polymorphism of the alleles of the merozoite surface antigens MSA1 and MSA2 in Plasmodium falciparum wild isolates from Colombia. Mol. Biochem. Parasitol. 49:265-275.

4. Thaithong, S., G.H. Beale, B. Fenton, J. McBride, V. Rosario, A. Walker, and D. Walliker. 1984. Clonal diversity in a single isolate of the malaria parasite Plasmodium falciparum. Trans. R. Soc. Trop. Med. Hyg. 78:242-245.

5. Babiker, H.A., A.M. Creasey, B. Fenton, R.A.L. Bayoumi, D.E. Arnot, and D. Walliker. 1991. Genetic diversity of Plasmodium falciparum in a village in eastern Sudan. 1. Diversity of enzymes, 2D-PAGE proteins and antigens. Trans. R. Soc. Trop. Med. Hyg. 85:572-577.

6. Le Bras, J., P. Deloron, A. Ricoor, B. Andrieu, I. Savel, and P. Coulaud. 1983. Plasmodium falciparum: drug sensitivity in vitro of isolates before and after adaptation to continuous culture. Exp. Parasitol. 56:9-14.

7. Viriyakosol, S., N. Siripoon, X.P. Zhu, W. Jarra, A. Seugorn, K.N. Brown, and G. Snounou. 1994. Plasmodium falciparum: selective growth of subpopulations from field samples following in vitro culture, as detected by the polymerase chain reaction. Exp. Parasitol. 79:517-525.

8. Bayoumi, R.A.L., A.M. Creasey, H.A. Babiker, J.M.-R. Carlton, A.A. Sultan, G. Satti, A.K. Sohal, D. Walliker, J.B. Jensen, and D.E. Arnot. 1993. Drug response and genetic characterization of Plasmodium falciparum clones recently isolated from a Sudanese village. Trans. R. Soc. Trop. Med. Hyg. 87: 454-458.

9. Arnot, D.E., C. Roper, and R.A.L. Bayoumi. 1993. Digital codes from hypervariable tandemly repeated DNA sequences in the Plasmodium falciparum circumsporozoite gene can genetically barcode isolates. Mol. Biochem. Parasitol. 61:15-24.

10. Mercereau-Puijalon, O., T. Fandeur, S. Bonnefoy, C. Jacquemot, and J.-L. Sarthou. 1991. A study of the genomic diversity of Plasmodium falciparum in Senegal. 2. Typing by use of polymerase chain reaction. Acta Trop. 49:293304.

11. Daubersies, P., S. Sallenave-Sales, J.F. Trape, L. Raharimalala, C. Rogier, H. Contamin, T. Fandeur, C. Daniel-Ribeiro, O. Mercereau-Puijalon, and P. Druilhe. 1994. PCR characterization of isolates from various endemic areas: diversity and turnover of Plasmodium falciparum populations are correlated with transmission. Mem. Inst. Oswaldo Cruz. 89:9-12.

12. Kimura, M., O. Kaneko, A. Inoue, A. Ishii, and K. Tanabe. 1995. Amplification by polymerase chain reaction of Plasmodium falciparum DNA from giemsa-stained thin blood smears. Mol. Biochem. Parasitol. 70:193-197.

13. Contamin, H., T. Fandeur, C. Rogier, S. Bonnefoy, L. Konate, J.-F. Trape, and O. Mercereau-Puijalon. 1996. Different genetic characteristics of Plasmodium falciparum isolates collected during successive clinical malaria episodes in Senegalese children. Am. J. Trop. Med. Hyg. 54:632-643.

14. Mazier, D., P. Druilhe, C. Guguen-Guillouzo, P. Bayard, V. Soeun, A. Datry, and M. Gentilini. 1984. Hepatocytes as feeder-layers for in vitro cultivation of Plasmodium falciparum blood-stages. Trans. R. Soc. Trop. Med. Hyg. 78:330-334.

15. Hill, D.R., R.H. Behrens, and D.J. Bradley. 1996. The risk of malaria in travelers to Thailand. Trans. R. Soc. Trop. Med. Hyg. 90:680-681.

16. Rosenberg, R., R.G. Andre, S. Ngampatom, C. Hatz, and R. Burge. 1990. A stable, oligosymptomatic malaria focus in Thailand. Trans. R. Soc. Trop. Med. Hyg. 84:14-21.

17. Segal, H.E., R.N. Wilkinson, W. Thiemanun, W.E. Gresso, and D.J. Gould. 1974. Longitudinal malaria studies in rural north-east Thailand: demographic and temporal variables of infection. Bull. WHO. 50:505-512.

18. Nosten, F., C. Luxemburger, D.E. Kyle, R. Ballou, J. Wittes, E. Wah, T. Chongsuphajaisiddhi, D. Gordon, N.J. White, J. Sadoff, and D.G. Heppner. 1996. Randomised double-blind placebo-controlled trial of SPf66 malaria vaccine in northwestern Thailand. Shoklo SPf66 Malaria Vaccine Trial Group. Lancet. 348:701-707.

19. Druilhe, P., P. Jacquier, D. Lambert, and M. Gentilini. 1980. Plasmodium falciparum in vitro culture: improvements using umbilical cord serum and medium modifications. Tropenmed. Parasitol. 31:409-413.

20. Brasseur, P., J. Kouamouo, O. Brandicourt, R. Moyou-Somo, and P. Druilhe. 1988. Patterns of in vitro resistance to chloroquine, quinine, and mefloquine of Plasmodium falciparum in Cameroon, 1985-1986. Am. J. Trop. Med. Hyg. 39:166-172.

21. Druilhe, P., P. Brasseur, O. Brandicourt, J. Kouamouo, D. Richard-
Lenoble, F. Diouf, F. Gay, R.S. Moyou, M. Danis, M.Y. Kombila, et al. 1986 Plasmodium falciparum drug resistance in West Africa. Ann. Soc. Belge Med. Trop. 66:297-300.

22. Fandeur, T., and O. Mercereau-Puijalon. 1991. Plasmodium falciparum: genetic stability of the Uganda Palo Alto strain propagated in the squirrel monkey (Saimiri sciureus). Exp. Parasitol. 72:223-235.

23. Van der Ploeg, L.H.T., M. Smits, T. Ponnudurai, A. Vermeulen, J.H.E.T. Meuwissen, and G. Langsley. 1985. Chromosome-sized DNA molecules of Plasmodium falciparum. Science. 229:658-661.

24. Contamin, H., T. Fandeur, S. Bonnefoy, F. Skouri, F. Ntoumi, and O. Mercereau-Puijalon. 1995. PCR typing of field isolates of Plasmodium falciparum. J. Clin. Microbiol. 33:944-951.

25. Patarapotikul, J., and G. Langsley. 1988. Chromosome size polymorphism in Plasmodium falciparum can involve deletions of the subtelomeric pPFrep20 sequence. Nucleic Acids Res. 16:4331-4340.

26. Sambrook, J., E.F. Fritsch, and T. Maniatis. 1989. Molecular Cloning: A Laboratory Manual, 2nd edition. Advances in Immunology. Cold Spring Harbor Laboratory Press, Plainview, New York.

27. Certa, U., D. Rotmann, H. Matile, and R. Reber-Liske. 1988. A naturally occurring gene encoding the major surface antigen precursor p190 of Plasmodium falciparum lacks tripeptide triplets. EMBO (Eur. Mol. Biol. Organ.) J. 6:4137-4142.

28. Tanabe, K., M. Mackay, M. Goman, and J.G. Scaife. 1987. Allelic dimorphism in a surface antigen gene of the malaria parasite Plasmodium falciparum. J. Mol. Biol. 195:273-287.

29. Smythe, J.A., M.G. Peterson, R. Coppel, A.J. Saul, D.J. Kemp, and R.F. Anders. 1990. Structural diversity in the 45-kilodalton merozoite surface antigen of Plasmodium falciparum. Mol. Biochem. Parasitol. 39:227-234.

30. Rosario, V. 1981. Cloning of naturally occurring mixed infections of malaria parasites. Science. 212:1037-1038

31. Brasseur, P., J. Kouamouo, R. Moyou-Somo, and P. Druilhe. 1992. Multi-drug resistant falciparum malaria in Cameroon in 1987-1988. II. Mefloquine resistance confirmed in vivo and in vitro and its correlation with quinine resistance. Am. J. Trop. Med. Hyg. 46:8-14.

32. Brasseur, P., J. Kouamouo, R. Moyou, and P. Druilhe. 1990. Emergence of mefloquine-resistant malaria in Africa without drug pressure. Lancet. 336:59.

33. Cowman, A.F., D. Galatis, and J.K. Thompson. 1994. Selection for mefloquine resistance in Plasmodium falciparum is linked to amplification of the pfmdr1 gene and cross-resistance to halofantrine and quinine. Proc. Natl. Acad. Sci. USA. 91:1143-1147.

34. Kemp, D.J., L.M. Corcoran, R.L. Coppel, H.D. Stahl, A.E. Bianco, G.V. Brown, and R.F. Anders. 1985. Size variation in chromosomes from independent cultured isolates of Plasmodium falciparum. Nature. 315:347-350.

35. Oquendo, P., M. Goman, M. Mackay, G. Langsley, D. Walliker, and J. Scaife. 1986. Characterization of a repetitive DNA sequence from the malaria parasite, Plasmodium falciparum. Mol. Biochem. Parasitol. 18:89-101.

36. Dolan, S.A., A. Herrfeldt, and T.E. Wellems. 1993. Restriction polymorphisms and fingerprint patterns from an interspersed repetitive element of Plasmodium falciparum DNA. Mol. Biochem. Parasitol. 61:137-142.

37. Goman, M., G. Langsley, J.E. Hyde, N.K. Yankovsky, J.W. Zolg, and J. Scaife. 1982. The establishment of genomic DNA librairies for the human malaria parasite Plasmodium falciparum and identification of individual clones by hybridization. Mol. Biochem. Parasitol. 5:391-400.

38. Kemp, D.J., A.F. Cowman, and D. Walliker. 1990. Genetic diversity in Plasmodium falciparum. Adv. Parasitol. 29:75-149.

39. Paul, R.E.L., M.J. Packer, M. Walmsley, M. Lagog, L.C. Ranford-Cartwright, R. Paru, and K.P. Day. 1995. Mating patterns in malaria populations of Papua New Guinea. Science. 269:1709-1711.

40. Pringle, G. 1966. A quantitative study of naturally-acquired malaria infections in Anopheles gambiae and Anopheles funestus in highly malarious area of East Africa. Trans. R. Soc. Trop. Med. Hyg. 60:626-632.

41. Ranford-Cartwright, L.C., P. Balfe, R. Carter, and D. Walliker. 1991. Genetic hybrids of Plasmodium falciparum identified by amplification of genomic DNA from single oocysts. Mol. Biochem. Parasitol. 49:239-244.

42. Walliker, D., I.A. Quakyi, T.E. Wellems, T.F. McCutchan, A. Szarfman, W.T. London, L.M. Corcoran, T.R. Burkot, and R. Carter. 1987. Genetic analysis of the human parasite Plasmodium falciparum. Science. 236:1661-1666.

43. Walliker, D. 1989. Genetic recombination in malaria parasites. Exp. Parasitol. 69:303-309.

44. Trager, W., M. Tershakovec, L. Lyandvert, H. Stanley, N. Lanners, and E. Gubert. 1981. Clones of the malaria parasite Plasmodium falciparum obtained by microscopic selection: their characterization with regard to knobs, chloroquine sensitivity, and formation of gametocytes. Proc. Natl. Acad. Sci. USA. 78:6527-6530.

45. Brasseur, P., J. Kouamouo, R. Moyou-Somo, and P. Druilhe. 1992. Multi-drug resistant falciparum malaria in Cameroon in 1987-1988. I. Stable figures of prevalence of chloroquine- and quinine-resistant isolates in the original foci. Am. J. Trop. Med. Hyg. 46:1-7. 